\title{
Studies in Optimal Configuration of the LTP
}

\author{
Wayne R. McKinney,* Mark Anders, Samuel K. Barber, Edward E. Domning, Yunian Lou, Gregory \\ Y. Morrison, Farhad Salmassi, Brian V. Smith, and Valeriy V. Yashchuk
}

Lawrence Berkeley Laboratory, Mailstop 2R0400, One Cyclotron Rd., Berkeley, CA 94720-8199

\begin{abstract}
Brightness preservation requirements for ever brighter synchrotron radiation and free electron laser beamlines require surface slope tolerances of x-ray optics on the order of $0.2 \mu \mathrm{rad}$, or better. Hence, the accuracy of dedicated surface slope metrology must be $0.1 \mu \mathrm{rad}$, or even less. Achieving this level of measurement accuracy with the flagship instrument at synchrotron radiation metrology laboratories, the Long Trace Profiler (LTP), requires all significant sources of systematic, random, and instrumental drift errors to be identified, and reduced or eliminated. In this respect, the performance of certain components of the Advanced Light Source LTP-II design [Kirschman, et al., Proc. SPIE, 7077, 70770A-12 (2008)] is analyzed, considering the principal justification for inclusion of each component, possible systematic error due to the quality of its optical material, and drift effects due to generated heat, etc. We investigate the effects of replacement of the existing diode laser with a fiber-coupled laser light source, and demonstrate that reducing the number of components by using a single beam on the surface under test (SUT), rather than an original double beam maintains, or even improves the accuracy of measurement with our LTP. Based on the performance of the upgraded LTP, we trace the further steps for improving of the LTP optical system.
\end{abstract}

Keywords: slope measurement, LTP, long trace profiler, optical metrology, x-ray optics, synchrotron radiation, beamline

\section{INTRODUCTION}

As the emittance of storage rings and free electron lasers falls with each new generation of machine or improvement to existing machines the requirement that the optical components not destroy the brightness and coherence of the $\mathrm{x}$-ray beams results in ever tighter specifications. $0.1 \mu \mathrm{rad}$ rms slope measurements over a spatial wavelength band from a few $\mathrm{mm}$ to the length of the optic become essential. Now classical arguments about the difficulties and expense of normal Fizeau interferometry for long narrow optics of often unusual figure shape have led to the construction of long trace profilers (LTP) at practically all synchrotron radiation laboratories ${ }^{1-8}$ There are many versions of the LTP, as implemented in synchrotron laboratories. Names run from LTP thru LTP II to LTP V. Mainly, they differ by type of air bearing and beam, type of angle measuring system - whether pencil-beam interferometer with a 1D or 2D detector, ${ }^{1,9}$ or autocollimator, and whether the entire measuring system or just a pentaprism moves over the optic along with the carriage. ${ }^{10-12}$

Before the improvements described in this article, we choose to define the upgraded ALS LTP, ${ }^{1}$ as an LTP II + . Our original LTP was made by a collaboration of Lawrence Berkeley Laboratory (LBNL), Brookhaven National Laboratory (BNL), Continental Optical, Fox Air Bearing, and Baker Engineering, in 1991/1992. Baker Engineering provided mechanical system design, Fox the air bearings, and BNL and LBNL the optical design. Continental Optical was the general contractor, and optical component supplier. The reference mirror was added for this version by Irick et al., traditionally designated the LTP II. ${ }^{2}$ A significant parallel LTP-like development occurred in Europe lead by Lammert ${ }^{7}$ resulted in the Nanometer Optische Messmachine (NOM). The basic NOM design has been copied many times, and is the basis for a new North American effort for a next generation LTP. ${ }^{13}$ LTP IV and LTP V were the designations of instruments built by Ocean Optics, the successor to Continental for commercial LTP manufacture.

We chose the designation LTP II+ because of the major improvements that we have made since it was first constructed; new LabView software controls, flat field calibrated 2D detector of smaller pixel size, stop-start motion, etc. ${ }^{1,14}$

*wrmckinney@,lbl.gov; phone 1-510-486-4395; fax 1-510-486-4385; http:/www-esg.lbl.gov/Production/OML/index.html 
Still, after these significant improvements our LTP II+ remains affected by several problems which limit the accuracy to be greater than $0.1 \mu \mathrm{rad}$. Random noise is mainly caused by air motion in the optical path, ${ }^{15}$ particularly the reference beam path which is long in the case of a long optic. Major systematic error is introduced by inhomogeneities in the glass of the prisms, lens, beam splitters, and microroughness on the surfaces of the folding mirrors as shown in particular, by Takacs and Qian. ${ }^{16}$ Temperature variation in our optical metrology laboratory (OML) affects the phase difference between the two beams, introducing false radii of curvature from slow drifts over the course of an entire scan, and periodic error from the cycling on and off of the heating/cooling system. The discrete nature of the CCD camera appears in errors of fitting a parabola to the classical LTP double peaked focused spot on the detector. ${ }^{17}$ This error is estimated at approximately 50 nanoradians in our LTP II+ setup.

\section{ANALYSIS OF PHASE SHIFT PROBLEMS OF THE LTP II+}

When making a measurement with the LTP II+ one adjustment stands out as necessary to be continually monitored during a scan. This is relative phase shift between the two beams which travel both to the surface under test (SUT) and the reference beam. Figure 1 shows a partial schematic diagram of the LTP II+. Laser light enters a non-polarizing beam splitter (BS) from the right, and passes through two roof prisms. The top prism, P1 is made moveable with a small mechanical slide and fine pitched screw. This mechanism provides two essential things for the LTP II+: adjustable separation between the two beams, typically $1-2 \mathrm{~mm}$, and adjustable phase difference between the two almost plane wavefronts of each beam. A phase shift of $180^{\circ}$ or $\pi$ radians is necessary for the two beams to have a double peaked destructive interference image pattern on the detector after being focused by the $\mathrm{f} /$ theta lens.

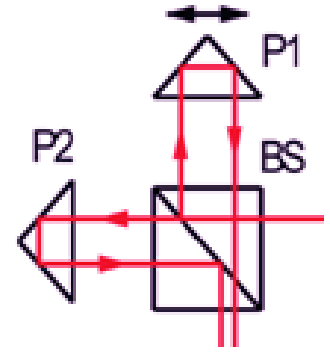

Figure 1. Partial schematic of the LTP II+ showing the laser beams, polarizing beam splitter, and roof prisms.
After transformation of the angle to distance by the $\mathrm{f} /$ theta lens the spot is essentially a convolution of Young's double slit fringes and an approximately Gaussian beam image. We assume that this arrangement was necessary in the original LTPs in order to provide a accurate way to find the center of the image. Lasers at the time were inherently more noisy, showing noise on top of a beam profile that may have not been completely Gaussian. Fitting about a dozen points of the convolved interference image to a parabola that passed through the minimum between the two peaks of the interference pattern has provided a satisfactory way to find the center of the image for more than two decades for most LTPs. In addition, problems from potential saturation of the detector were avoided by the use of the destructive interference pattern in the middle.

Instability of the phase shifter of the LTP II+ (Fig. 1) is the major impediment to implementation of the recently developed methods for reduction of the errors due to systematic effects and instrumental drifts. ${ }^{1,10,18}$ These methods are based on averaging of multiple measurements performed at different experimental arrangements that requires a significant time of stable operation of the instrument. The problem of instability can be solved in two radical ways. First, one can use another, more stable, optical schematic to provide the phase shift - Sec. 3. Alternatively, the shifter can be totally removed for LTP operation under conditions without destructive interference (Gaussian spot on the detector Sec. 6.)

\section{PHASE SHIFTING PLATE}

In the manner of Zhao et al., ${ }^{19}$ and Qian and Takacs ${ }^{20-22}$ we have constructed a stepped phase plate which allows the potential for eliminating all of the components shown in Fig. 1, and replacing them with an optical window with a $\lambda / 2$ step. ${ }^{23}$ Figure 2 shows the phase shifter plate. It was made on an high quality fused silica window. Sputtered $\mathrm{SiO}_{2}$ was added in a smooth layer using a single edged razor blade as a mask. The design includes a $10^{\circ}$ tilt angle for the plate to allow some adjustment around the nominal value, to allow tolerance in manufacturing, and to eliminate spurious effects due to back reflections and interference. 


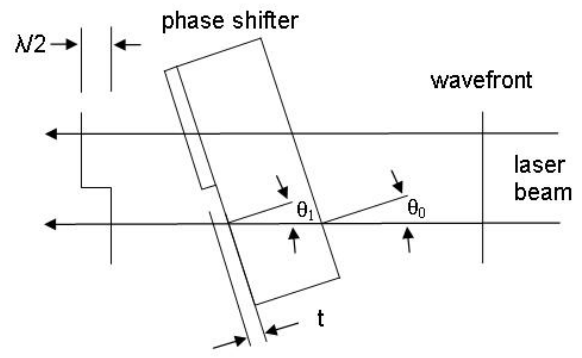

Figure 2. Operation of the phase shifter plate. The nominal tilt angle allows for adjustment, compensation of possible error in fabrication, and elimination of spurious effects due to back reflections and interference.

For a laser in the LTP with the wavelength of $\sim 676.4 \mathrm{~nm}$, the required difference in the optical path would be:

$$
\mathrm{OPD}=\lambda / 2=676.4 / 2 \mathrm{~nm}=338.2 \mathrm{~nm} \text {. }
$$

This can be converted to the required step height using Snell's Law. If the sputtered silica has refractive index $\mathrm{n}$, the optical path difference $p$, caused by the step height $t$, as a function of incidence angle $\theta$ is:

$$
\mathrm{OPD}=\mathrm{t}\left[\frac{\mathrm{n}}{\cos \left(\theta_{1}\right)}-\frac{1}{\cos \left(\theta_{0}\right)}\right]
$$

Three separate types of tests were performed on the fabricated part. First, a direct measurement of the induced phase shift was conducted in a simplified setup, similar to the configuration in the LTP. Additionally, the optical path difference was measured, as well as the physical step height. These two pieces of information allowed the refractive index of the sputtered silica (which was not known a priori) to be calculated from Eq. (2), which in turn allowed the phase shift to be calculated using;

$$
\varphi=\frac{2 \pi(\mathrm{OPD})}{\lambda},
$$

and compared to the measured value from the first test. The necessary thickness was determined to be $749.3 \mathrm{~nm}$. Knowledge of the physical step height also allowed adjustments to be made to the sputtering process. Note that some of the data presented below represent a part made earlier in the development process which had smaller phase shift than $180^{\circ}$.

\subsection{Phase Shift Measurements}

Figure 3 schematically shows a simple test system that was built to test the amount of phase shift induced by the plate. All the components before the phase shifter: the fiber collimator optics, neutral density filters (NDFs), polarizers (P1 and P2), and Polarizing Beam Splitter (PBS), were mounted on a sturdy support to reduce instability, consisting of all the aforementioned components on a ThorLabs ${ }^{\mathrm{TM}}$ cage system, mounted on a pair of posts to the optical table.

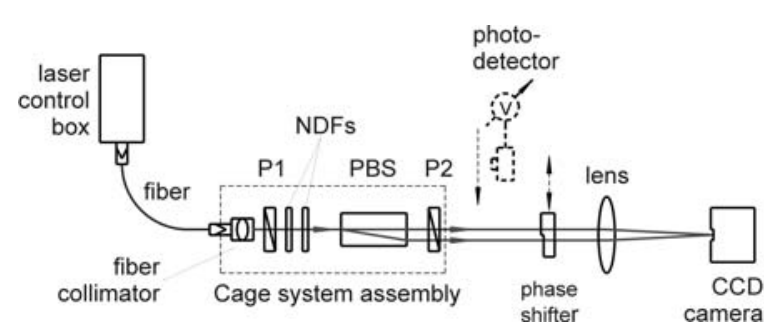

Figure 3. Schematic of phase shift test configuration.
The first polarizer was used to fix the light polarization direction. Therefore, the instability of the polarization due to the used single-mode fiber was minimized. The NDFs allowed attenuation of the light intensity to a level optimal for interference pattern detection with the CCD camera. A calcite beam splitter provided the two beams of a pencil beam interferometer. However, because the beams after the PBS were orthogonally polarized, an additional polarizer, $\mathrm{P} 2$, was used, oriented with its main axis at approximately 45 degrees with respect to the axis of polarization of the PBS.

Besides providing the same linear polarization of the output beams, the $2^{\text {nd }}$ polarizer provided additional balancing of the beam intensities. A ZYGO spherical reference lens with a focal distance of $722 \mathrm{~mm}$ was used to overlap the beams in the focal plane, where a CCD camera was placed to record the interference patterns. In the course of balancing, a removable ThorLabs $^{\mathrm{TM}}$ photo-detector (Fig. 2) was placed between the cage system assembly and the lens to monitor relative intensities of the beams. A paper pipe was also placed around the beams between the lens and the CCD camera to reduce 
fluctuations in the optical path due to air convection. To further reduce these fluctuations, the enclosure around the optical table was left closed long enough for any major temperature distributions in the enclosure to dissipate.

Before any readings were taken, the alignment of the phase shifter was calibrated to ensure that each beam passed cleanly to either side of the step on the phase shifter, and the polarizers were adjusted so that the beams were as equal in intensity as possible. Profiles of the interference pattern on the CCD were then recorded without the phase shifter until the system had settled to a relatively constant state. Data was then taken with and without the phase shifter in the optical path. In order to additionally reduce spurious effect due to the set-up drifts, multiple (up to 35 total) measurements were done at a time, alternating with and without the phase shifter in sets of five measurements in each state. The resulting intensity profiles were then exported to be analyzed-Fig 4 . Additionally, measurements were taken with both beams on the untreated side and both beams on the sputtered side of the phase shifter, to test the uniformity of the glass and ensure that the induced phase shift was due only to the step difference between the two sides of the phase shifter. The total procedure was repeated multiple times to ensure reproducibility. Note that the incidence angle dependence of the OPD of the ordinary and extraordinary beams of a calcite beam splitter is currently under analysis. A preliminary result of the analysis has proved that the dependence is very strong. The goal of the analysis is to verify whether or not such a PBS can be used as a basic sensor element for slope measuring.

To extract the phase difference caused by the phase shifter, a model fitting function that is a result of convolution of two beams with a Gaussian intensity distribution and with an arbitrary relative phase shift ${ }^{3,24}$ was used to fit the measured interference pattern data-Fig 5. The resulting interference profile is a Gaussian distribution modulated by a cosine:

$$
I(x)=A e^{-B\left(x-x_{0}\right)^{2}}\left[1+C \cos \left(D\left(x-x_{0}\right)+\phi\right)\right]+E .
$$

This model describes the intensity as a function of position in terms of seven parameters:

\begin{tabular}{|l|l|}
\hline A: & governs the overall amplitude of the intensity distribution; \\
\hline B: & determines the width of the Gaussian distribution and depends on the beam diameter; \\
\hline C: & controls how close to zero the dark fringes are; \\
\hline D: & changes the frequency of the cosine to set the fringe separation; which correlates with the beam separation; \\
\hline E: & offset, the CCD has a threshold value; seen by the sharp corners at the edges of the measured patterns in Fig. $3 ;$ \\
\hline x0: & locates the center of the interference pattern; \\
\hline$\varphi:$ & phase shift between the beams; when perfectly in phase the pattern should be at a maximum at $\mathrm{x}=\mathrm{x} 0$ and $\varphi=0$. \\
\hline
\end{tabular}

The first six parameters have the physical meaning as pointed out above. They are to be determined and fixed as constants. These parameters, which depend entirely on the geometry of the system, specifically the center of the interference pattern and the spacing of the fringes, were found to deviate by no more than $1 \%$ over the entire set of measurements. Thus, the average values for these parameters were taken as constants in the model and a regression analysis was run again with fewer parameters, allowing for an accurate determination of the phase parameter.

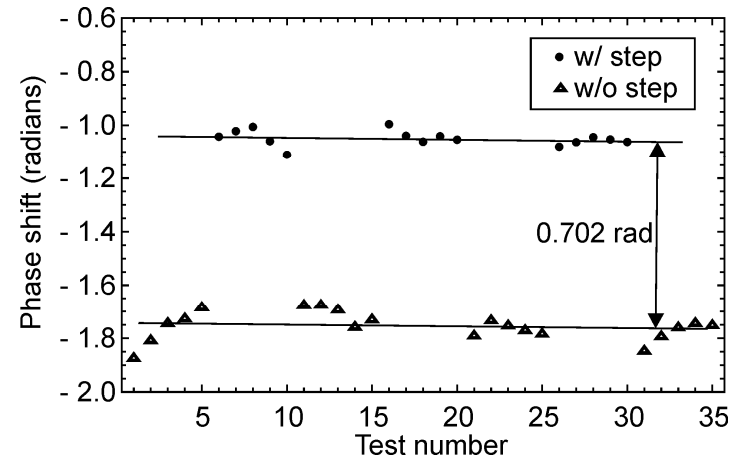

Figure 4. Measured interference pattern vs. the fitted model, with and without phase shifter. Early developmental part shifts the phase by approximately $43^{\circ}$. Each point is a result of fitting an interference pattern (Fig. 5).

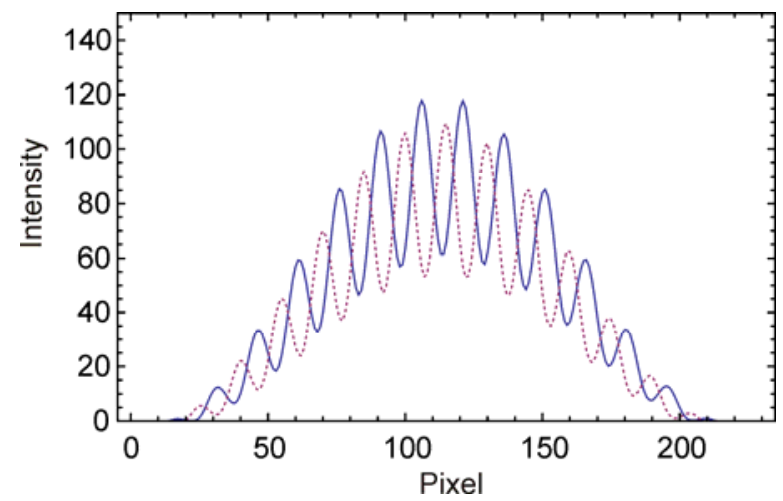

Figure 5. Interference patterns of final prototype phase shifter with phase shifter (dashed lined) and without phase shifter (solid line). Final prototype produces approximately 180 degree phase shift. 


\subsection{Optical Path Difference Measurements Zygo GPI ${ }^{\mathrm{TM}}$ interferometer}

In addition to direct measurements of phase shift, it was desirable to measure the optical path difference OPD (see Eq. 1) between the two sides of the phase shifter. This was done by mounting the phase shifter between a high quality plane reference mirror and a Zygo interferometer, which reads the optical path difference over the step.
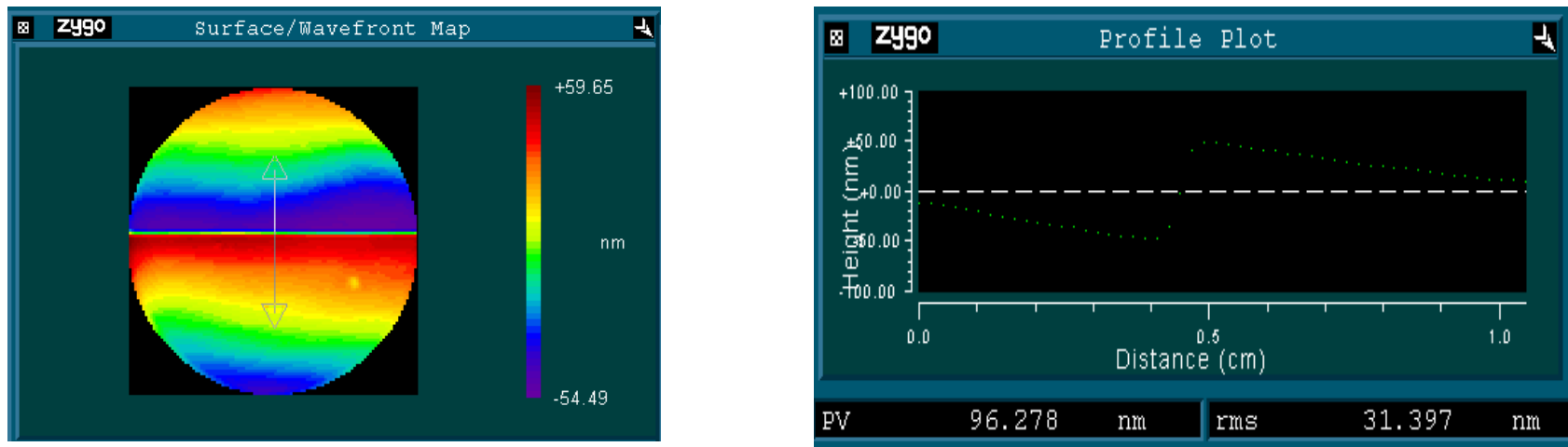

Figure 6 Sample wavefront map and profile plot from Zygo interferometer for the $43^{\circ}$ prototype part. The spot in the lower right quadrant is due to dust on the optics.

Figure 6 shows the wavefront map of light passing through the phase shifter and a profile over the step. The apparent tilt of the sample is due to the Zygo software averaging the values about zero. The resulting angle of the step is not significant since the scales of the $\mathrm{X}$ and $\mathrm{Y}$ axes are different by many orders of magnitude. Such profiles were taken along the length of the step in increments of about $1 \mathrm{~mm}$. The average peak-to-valley difference in optical path was $96.87 \mathrm{~nm}$, with values ranging from $94.9 \mathrm{~nm}$ to $101.4 \mathrm{~nm}$. Note that the measured OPD is due to two passes of the ZYGO light beam through the phase shifter. As with the direct phase shift measurements in Sec. 3.1, the uniformity of the glass on either side of the step was also measured. The sputtered and untreated sides of the prototype phase shifter had an rms roughness in their optical path of $2.3 \mathrm{~nm}$ and $1.0 \mathrm{~nm}$, respectively.

\subsection{Step Height Measurements with Micromap ${ }^{\mathrm{TM}}-570$ interferometric microscope}

The third test performed on the phase shifting plates was a measurement of the physical step height, using an interferometric microscope. The height was measured in $1 \mathrm{~mm}$ increments along the step, which could be compared to the $1 \mathrm{~mm}$ increment measurements with the interferometer. Fig. 7 shows a sample measurement taken with the microscope. Note that the relatively small focusing depth of the microscope allows a measurement of the topography of the face up step surface without perturbation due to the light reflected from the bottom surface.

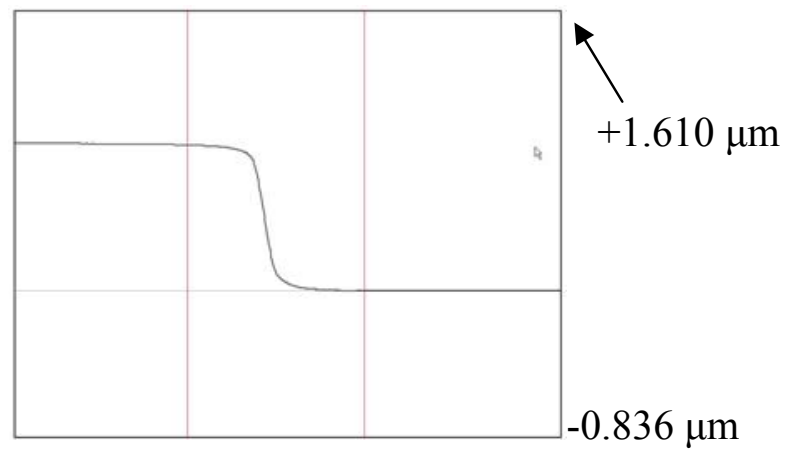

Figure 7. Sample result from the MicromapTM-570 interferometric microscope step height measurement, $\sim 180^{\circ}$ part.

\subsection{Test Results and Discussion}

The sputtering process has been shown to provide a step thickness that is very close to the intended target value. Using the derived refractive index $n$ from the part which gave a $43^{\circ}$ phase angle, and solving Eq. (3) for $t$, the required step
The step height on one of the first prototypes averaged over a number of measurements across the step was $212.3 \mathrm{~nm}$, with values ranging from $209.6 \mathrm{~nm}$ to 214.9 nm. Using the measured values for optical path difference $p$ and step height $t$, the refractive index of the sputtered silica was calculated using Eq. (2): $n=1.4563$. This value of the refractive index of the sputtered silica is very close to that of fused silica. This is an important result of the measurement that allowed us to more reliably specify the step thickness of the final phase shifter for the LTP.

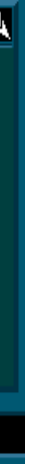


height was calculated to be, $t \sim 749.3 \mathrm{~nm}$, assuming a 10 degree incidence angle. We have fabricated 3 phase shifters with shifts close to $180^{\circ}$, but have not tested them in the LTP due to time constraints, and the fact that using just a single beam for the reference and surface under test has proven to provide the required accuracy and stability, see below. We hold the phase plate beamsplitter in reserve for the potential case that our single beam implementation has problems that would be addressed by doubling the beams. For the fabrication of a production phase shifter with a best chosen correct height, it may be favorable to err on the side of sputtering too little rather than too much, since the phase shift can only be increased slightly by increasing the tilt angle, but not decreased further if the phase shift is too high at a tilt angle close to zero.

\section{LTP LIGHT SOURCE BASED ON A FIBER COUPLED DIODE LASER}

\subsection{Pointing instability}

Previously, Yashchuk et al. have demonstrated ${ }^{15}$ that a fiber coupled diode laser with an output collimating lens showed less noise from pointing instability in a setup with a geometry similar to the LTP II+ than did the LTP II+ itself. This setup used a Nanoscan ${ }^{\mathrm{TM}}$ beam profiler to precisely measure the position of the laser spot at a distance of about one meter, similar to the distance to and from the reference mirror in an LTP. In this experimental arrangement, the question of laser pointing instability is co-mingled with the pointing error due to air convection along the optical path, as well as due to temperature dependence of the laser assembly. ${ }^{25-26}$ Placing the diode laser in a closed box, removed from the LTP head, and bringing the beam into the head via a single mode, polarization preserving fiber has the advantage of removing the heat generated by the laser from the head. The fiber works as a spatial filter, guaranteeing that the pointing instability of the diode laser itself does not effect the direction of the output beam. However, the possibility that the motion of the carriage which will continuously bend the fiber placed in the flexible cable tray may affect the output polarization of the output beam, and hence affect the LTP measurement must be checked, see Sec. 4.2.

First we performed a check of the pointing stability of the fiber coupled diode laser (model \# SRT-F670S-4/APM from Micro Laser Systems, Inc.) using the Nanoscan ${ }^{\mathrm{TM}}$ beam profiler to establish the pointing and intensity stability of the laser system itself. The laser was placed at a distance of approximately $1 \mathrm{~m}$ from the Nanoscan ${ }^{\mathrm{TM}}$ aperture. The centroid positions in the vertical and horizontal directions were sampled at a rate of $5 \mathrm{~Hz}$ over a period of about 75 minutes. During this time the air conditioner was switched off to avoid the appearance of $\sim 15$ min periodic oscillations typical of our system. Consequently, the centroid temporal dependences exhibit a linear drift due to changes in ambient temperature, which affects the mechanical stability of the experimental setup. This linear drift is subtracted from the data below to focus on the stability of the laser itself. Figure 8 shows the temporal dependence of the angular deviation of the beam's centroid position in the vertical direction.
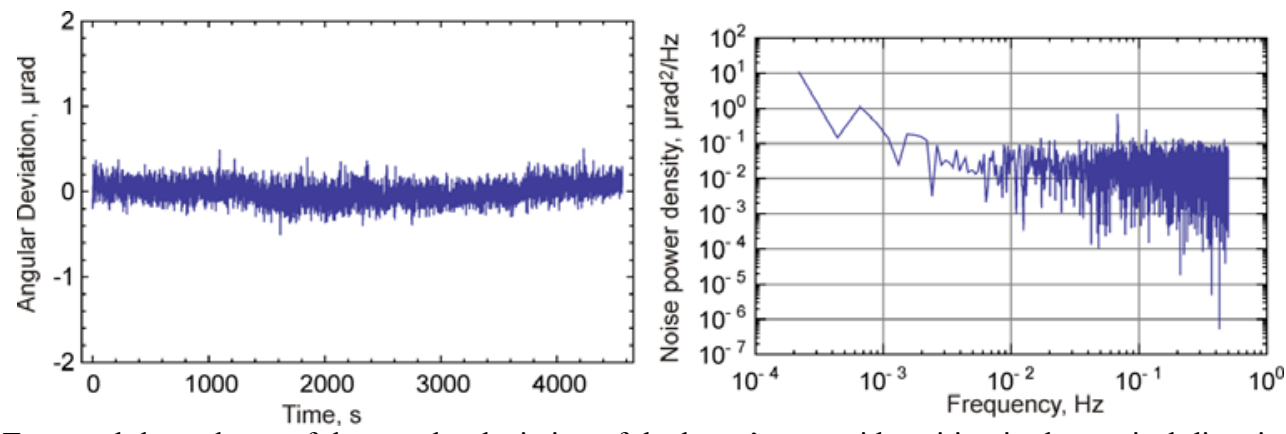

Figure 8. Temporal dependence of the angular deviation of the beam's centroid position in the vertical direction - the lefthand-side plot. Noise power density of the temporal dependence with white noise like character demonstrates the long term stability of the laser - the right-hand-side plot.

Before analyzing the data, each adjacent set of 5 data points was averaged to reduce the influence of the beam profiler noise. The RMS variation of this data is $0.14 \mu \mathrm{rad}$. In Fig. 8 the noise power density (NPD) spectrum of the measured time dependence is also shown. The NPD spectrum demonstrates the white noise like character of the pointing stability down to frequencies as low as $0.003 \mathrm{~Hz}$. At lower frequencies there is larger variation, as evidenced by the low frequency tail in the NPD, but these are most likely due to thermal variations in the room and not inherent to the laser output optical assembly. The contribution of these low frequency variations to the pointing instability can be removed by integrating the NPD over an appropriate bandwidth, which is $0.003 \mathrm{~Hz}$ to $0.5 \mathrm{~Hz}$. (Note that in the LTP II+, we average 10 sequential CCD camera frames collected during approximately 1 second) Over this bandwidth, the RMS variation of 
the centroid's angular position is on the order of $0.10 \mu \mathrm{rad}$, which is the desired limit for our purposes. Furthermore, the white noise like character of the NPD suggests an even greater stability can be achieved by simply averaging more consecutive scans. Note that only the pointing stability in the vertical direction has been discussed, but the pointing stability in the horizontal direction is nearly identical.

\subsection{Fiber bending tests}

As we have mentioned above, in the course of LTP scanning, a long fiber placed in the LTP flexible cable tray is a subject of continuous change of the place of $180^{\circ}$ bending. For this to work, however, it is critical that the parameters of the output light, such as intensity and polarization, stay reasonably stable. Conventional fiber optics can suffer from light intensity leaks through the fiber sidewalls and stress-induced birefringence as the fiber is bent, changing the intensity and the polarization of the output light.

With a conventional single-mode fiber, upon bending the fiber, it was immediately clear that this type of fiber was unsuitable for our requirements. The polarization changed unpredictably and by as much as 90 degrees with the slightest movement.

The polarization maintaining (PM) fiber showed much more potential, so in order to further test its performance, a balanced polarimeter set-up, shown in Fig. 9, was used. Since light can pass through the PM fiber in either direction, the experimental configuration was such that the laser with a control box was mounted on the movable LTP carriage, while the polarimeter was built onto the table (where the laser would ultimately be placed), to avoid mounting too much hardware onto the delicately-balanced carriage. The fiber was secured (as it would be in real-life use) inside the flexible cable tray that connects the carriage electronics to the rest of the system.

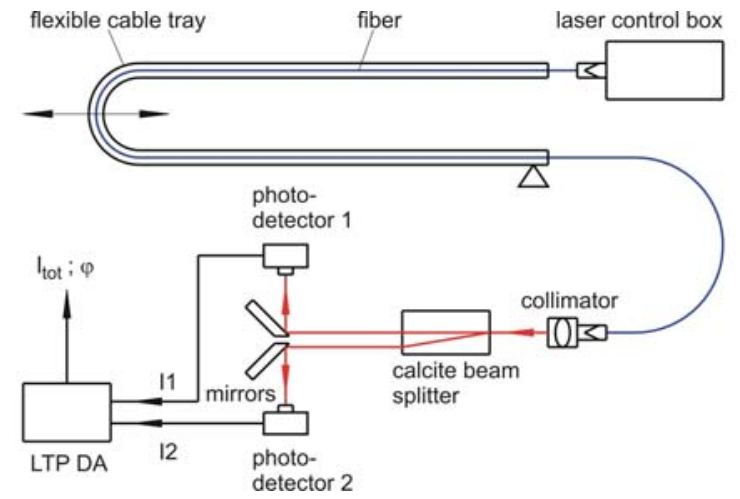

Figure 9. Schematic of the experiment based on a balanced polarimeter.
One end of the fiber was connected to the laser control box and the other end to a collimator. The collimator was placed on a rotating mount used for initial alignment of the output light polarization. A ThorLabs $^{\mathrm{TM}}$ calcite beam splitter was used as the polarizing beam splitter of a balanced polarimeter. The extinction ratio of the splitter is specified to be $>10^{5}$. The light polarization was aligned at 45 degrees with respect to the direction of the major axis of the splitter crystal. Alignment was made by balancing the light intensities of the split beams. Two light beams exiting the splitter have mutually orthogonal polarizations. As the polarization of the incoming light changes, the relative intensities of the two beams, $I_{1}$ and $I_{2}$, measured with the dedicated photo-detectors, also change. The normalized difference of the intensities is a measure of the rotation angle of the light polarization.

The conclusion from the performed measurements is that the tested polarization preserving 6-m-long fiber is adequate for use in the LTP. The observed variation of the polarization of $6^{\circ}$ does not significantly perturb balance intensities between the LTP sample and reference channels. The bending radius was $\sim 9 \mathrm{~cm}$. Moreover, if an additional linear polarizer is used after the fiber collimator, the polarization variation would only lead to a relatively small change of the overall output light intensity rather than changing the balance between the LTP channels. Details of the qualification experiments on the optical fiber may be obtained in Ref. ${ }^{27}$.

\subsection{Beam splitter}

We ordered 4 beamsplitter cubes, 2 polarizing (P) with associated 1/4 wave plates, and two non-polarizing (NP). All four were compared for transmitted wavefront error in all directions by measurement in an optical cavity of our 6-inch $\mathrm{Zygo}^{\mathrm{TM}}$ GPI interferometer and a good quality optical flat. The NP beamsplitters showed approximately a factor of 2 longer best-fit effective (two light passes) radii of curvature on outgoing wavefronts than the P beamsplitters. We chose to go with a NP beamsplitter, avoiding the extra elements of two $1 / 4$ wave plates, and one $1 / 2$ wave plate. ${ }^{28}$ While this makes the intensities between the two channels more difficult to adjust, we felt that the increase in optical quality, and less number of components justified the choice. 


\section{LTP II++ OPTICAL SCHEMATIC}

Figure 10 shows detailed models of the before (b) and after (a) optical designs. The new optical design utilizes a temperature stabilized, fiber coupled diode laser system, model \# SRT-F670S-4/APM with a 6-meter-long polarization maintaining fiber from Micro Laser Systems, Inc. The laser with a power supply and a temperature stabilizing circuit is placed on the LTP optical table. The output collimating optics are the only parts mounted on the carriage. The fiber placed in the LTP flexible cable tray passes the light between the laser and the collimator. As it was discussed above, ${ }^{1}$ this allows the solution of the heat and pointing instability problems of the on-carriage-mounted diode laser. As a result, the new system is able to provide a pointing stability on the level of $\sim 0.1 \mu \mathrm{rad}$ for the many hours necessary for multiple slope trace measurements with the LTP (see Sec. 6).

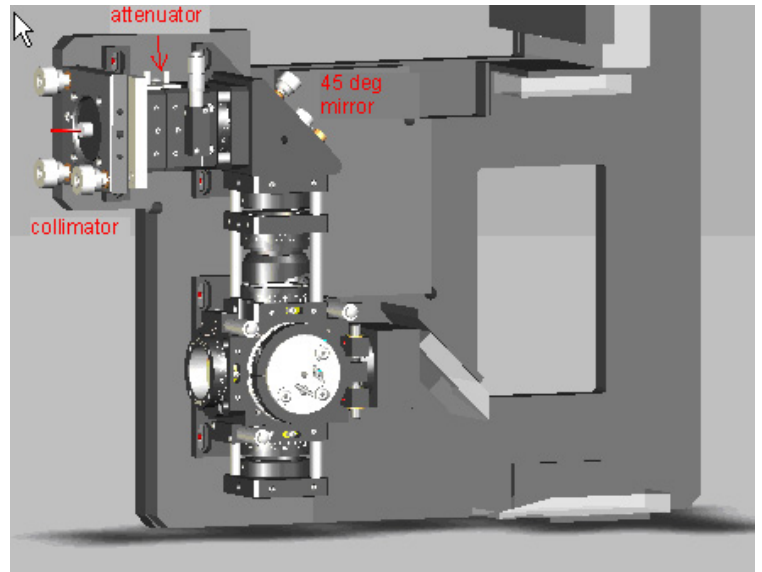

a LTP II++

Figure 10. a: New optical arrangement, LTP II++,

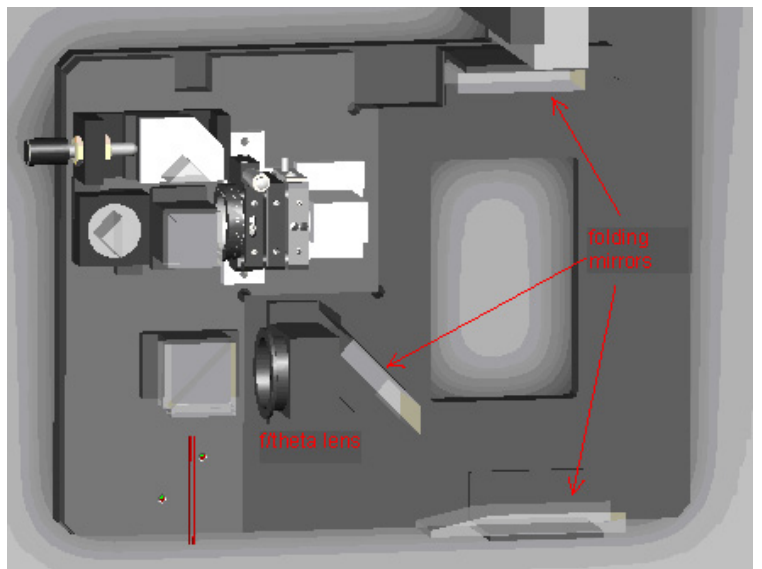

b LTP II+

b: older LTP II+ optical arrangement. ${ }^{1}$

The original LTP II+ had all of the optics mounted on two plates. We replaced the plate on the left hand side, simultaneously replacing all of the optics on the plate, conserving the old plate and optics for a potentially quick retrofit. Major points of the new optics were ability to replace components easily, use of easily available OEM mechanical parts, modular design, and stability due to fewer part count and mechanisms. These allow continuing R\&D work on improvement of the LTP optical sensor. The three folding mirrors and the f/theta lens remain in place, and were not modified, eliminating the need for angular re-calibration of the LTP.

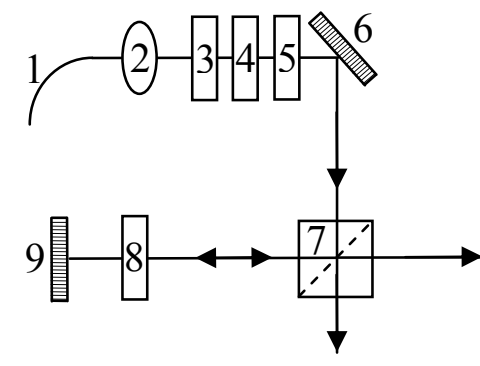

Figure 11. Optical schematic of the upgraded section of the LTP optical sensor system. Figure key presented to the right.

\begin{tabular}{|l|l|}
\hline Part & Function \\
\hline 1 & single mode polarization preserving fiber \\
\hline 2 & collimating lenses \\
\hline 3 & neutral density filter \\
\hline 4 & linear polarizer \\
\hline 5 & linear polarizer \\
\hline 6 & $45^{\circ}$ folding mirror \\
\hline 7 & non-polarizing beam splitter \\
\hline 8 & neutral density filter \\
\hline 9 & reference mirror \\
\hline
\end{tabular}

Notice that we have removed the Dove prism. Since, in principle, we have greatly reduced any possible laser pointing instability it is not needed since its purpose in the LTP II is to force the reference subtraction and laser pointing correction to have the same sign. ${ }^{29}$ Our initial testing has shown that the reference beam signal must be added in our configuration. This corrects the carriage pitch, but not periodic errors from thermal cycling of the room temperature. The removal of the Dove prism may need to be revisited. Another solution under investigation is to add one more reference channel for direct monitoring the light pointing instability. 


\section{PERFORMANCE TESTS WITH THE UPGRADED LTP II++}

\subsection{Stability test}

First, we performed a stability test with the new LTP optical sensor system. To carry out such a test we directly mounted a 1 in. gold coated mirror with a flatness of $\lambda / 20$ to the LTP optical head in the sample channel. With the test mirror attached to the LTP's translational carriage, any wobbling or other spurious motion of the carriage is effectively decoupled from the measurements in the sample channel. Thus, the measurements in the sample channel represent the stability of the laser in combination with the optical elements of the new LTP optical head during a typical measurement. For this test we performed a typical eight scan measurement ${ }^{18}$ over a scanning range of $210 \mathrm{~mm}$. The results of this measurement, shown in Fig. 12, are used to interpret how the new optical head's instability affects measurements in terms of curvature and RMS slope variation. That is, we can determine a typical contribution to measurements of curvature and RMS slope variation resulting from the instability of the new optical head. These contributions have been found to be extremely small, with an effective radius of curvature of $900 \mathrm{~km}$ and RMS slope variation of $0.05 \mu \mathrm{rad}$, leading to the conclusion that the new optical head is indeed very stable. These results can be thought of as the limiting accuracy of measurement for a flat optic.

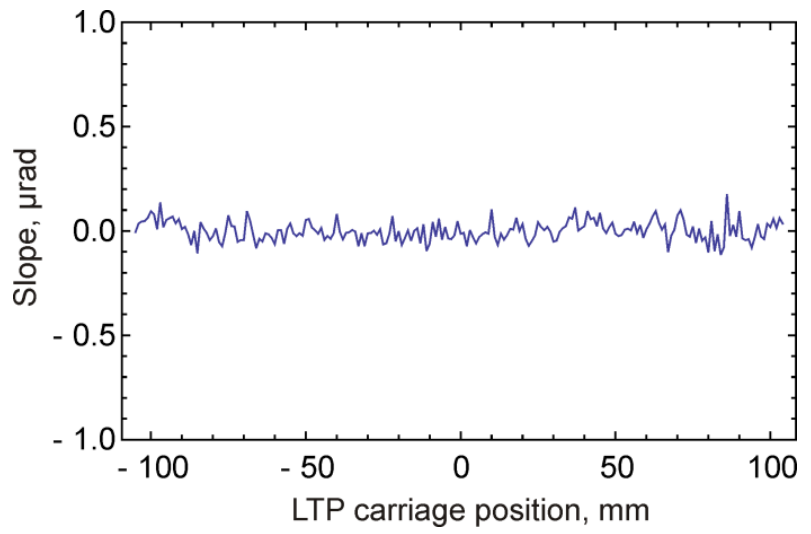

Figure 12. With the new LTP optical head installed, a stability scan was performed with a flat SUT attached to the LTP carriage. It was found that the instability of the new system introduces spurious curvature on the order of $900 \mathrm{~km}$ and a RMS slope variation on the order of $0.05 \mu \mathrm{rad}$, both of which are very small.

\subsection{Tests with a $40 \mathrm{~m}$ radius optic}

In order to characterize the performance of the new LTP configuration we compare measurements of a $40 \mathrm{~m}$ radius optic $^{30}$ made before and after the upgrade. This particular optic with length of $\sim 410 \mathrm{~mm}$ is suitable for a full characterization over the entire measurement range of the LTP.

For each optical head, the $40 \mathrm{~m}$ optic was measured using a typical eight scan measurement strategy. ${ }^{18}$ The residual slope traces from these measurements are shown in Fig. 13.

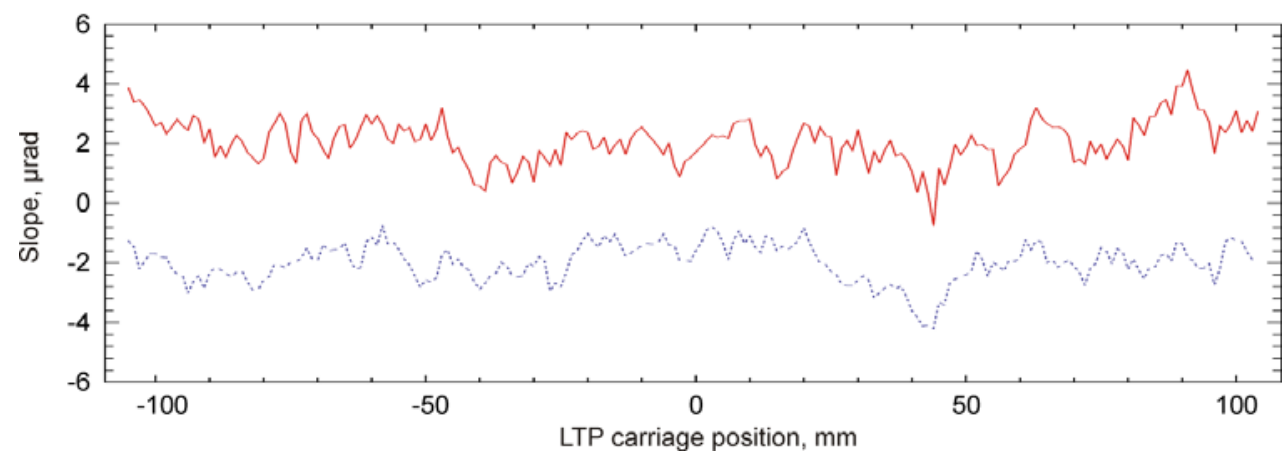

Figure 13. Residual slope traces of the $40 \mathrm{~m}$ reference optic using the old LTP optical head (dashed line) and the new optical head (solid line). In both cases an overnight eight scan measurement was performed. The radii of curvature for the two measurements are $40.240 \mathrm{~m}$ and $40.239 \mathrm{~m}$ for the old and new optical heads, respectively. The RMS slope variations are 0.64 $\mu \mathrm{rad}$ and $0.75 \mu \mathrm{rad}$, respectively. Offset is introduced. 
The measured radius of curvature was $40.240 \mathrm{~m}$ and 40.239 for the old and new optical heads, respectively. The RMS slope variation was $0.64 \mu \mathrm{rad}$ and $0.75 \mu \mathrm{rad}$ for the old and new optical heads, respectively. The radius measurements are in excellent agreement, but the RMS slope variation when using the new optical head is about $15 \%$ higher. An increase in the RMS slope variation might normally indicate a worse performance of the instrument, but in this case, the increase in RMS variation has to do with an increase in resolution with the new optical head. With the new optical head, the sample beam has a relatively small spot size with a narrow profile. Compared with the old optical head, there is an improvement in resolution of about 50\%. An improved resolution naturally leads a higher RMS variation as higher spatial frequency features are resolved. Direct measurements of the LTP II++ spatial resolution in the manner discussed in Siewert et al. and Rose et al. are planned. ${ }^{31-32}$

There is an additional way to demonstrate the improvement of the upgrade. Each measurement (with the old or new optical head) of the $40 \mathrm{~m}$ optic consisted of eight individual traces of the surface. These scans are taken in an appropriate sequence to average out long term drifts. ${ }^{18}$ By subtracting the average surface (average of the eight traces) from the individual traces in any given measurement, the instability inherent to any single trace is determined. Figure 14 shows the individual traces of each measurement with the respective average surface subtracted. In the case of Fig. 14, only the data from the sample channel was considered, i.e. there was no removal of the reference channel.
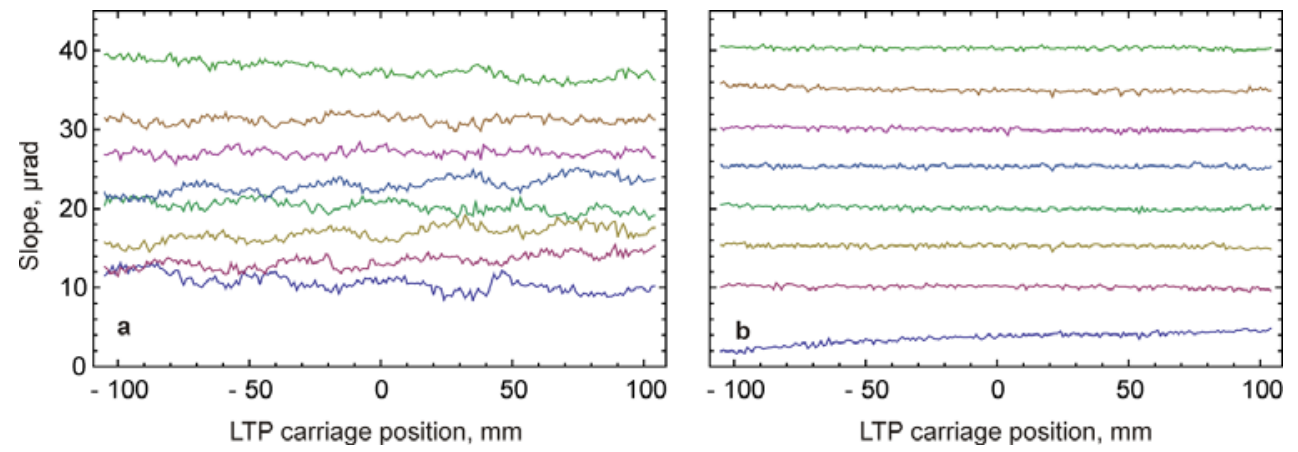

Figure 14. Individual sample channel traces from measurements of the $40 \mathrm{~m}$ optic made with the old LTP II $+(\mathrm{a})$ and new (b) LTP II++ optical heads. The respective average surface is subtracted from each trace. The data used in these figures do not include subtraction/addition of the reference channel. Offsets are added for better presentation.

\section{CONCLUSION AND NEXT STEPS}

The data presented in Fig. 14 definitively demonstrates the superior performance of the new LTP optical head compared to the old optical head. It is clearly seen that measurements made with the new optical head are almost completely insensitive to any long term drifts whereas there are major variations in the traces from the old optical head. The RMS variations for the traces in Fig. 14b are typically on the order of $0.2 \mu \mathrm{rad}$, whereas the RMS variations for the traces in Fig. 14a are on the order of $0.7 \mu \mathrm{rad}$. The combination of the fiber coupled diode laser and the simplified optical schematic has produced a significant improvement with regards to measurements made with the LTP. Since the entire impetus of this upgrade was to improve the long term stability of the LTP, this provides the most important result.

We see the proper steps to proceed to sub $0.1 \mu \mathrm{rad}$ absolute tangential slope measurement accuracy to be basically the following. The excellent temperature stability achieved in the HBZ/BESSY NOM setup, ${ }^{12,33-34}$ and the high thermal mass of the NOM have been widely recognized as crucial to the next generation of slope measuring instruments. A series of meetings of the North American metrology community has led to a draft specification of a new optical slope measuring system. ${ }^{13}$ Once all known improvements are in place, calibration of systematic error will become the limit. This will be addressed by the joint US-European project, the Universal Test Mirror (UTM). ${ }^{35-36}$ After this, newer detectors of smaller pixel size should permit a shorter focal length FT lens to be used, allowing the elimination of folding mirrors which are a significant source of systematic error from their microroughness and figure error. 


\section{ACKNOWLEDGEMENT}

The authors are grateful to Peter Takacs for very useful discussions. The Advanced Light Source is supported by the Director, Office of Science, Office of Basic Energy Sciences, Material Science Division, of the U.S. Department of Energy under Contract No. DE-AC02-05CH11231 at Lawrence Berkeley National Laboratory.

\section{DISCLAIMER}

This document was prepared as an account of work sponsored by the United States Government. While this document is believed to contain correct information, neither the United States Government nor any agency thereof, nor The Regents of the University of California, nor any of their employees, makes any warranty, express or implied, or assumes any legal responsibility for the accuracy, completeness, or usefulness of any information, apparatus, product, or process disclosed, or represents that its use would not infringe privately owned rights. Reference herein to any specific commercial product, process, or service by its trade name, trademark, manufacturer, or otherwise, does not necessarily constitute or imply its endorsement, recommendation, or favoring by the United States Government or any agency thereof, or The Regents of the University of California. The views and opinions of authors expressed herein do not necessarily state or reflect those of the United States Government or any agency thereof or The Regents of the University of California.

\section{REFERENCES}

[1] Kirschman, J. L., Domning, E. E., McKinney, W. R., Morrison, G. Y., Smith, B. V., and Yashchuk, V. V., "Performance of the upgraded LTP-II at the ALS Optical Metrology Laboratory," Proc. SPIE 7077, 70770A-12 (2008).

[2] Irick, S. C., McKinney, W. R., Lunt, D. L. J., and Takacs, P. Z., "Using a straightness reference in obtaining more accurate surface profiles from a long trace profiler," Rev. of Sci. Inst., 63, 1436-1438 (1992).

[3] von Bieren, K., "Pencil Beam Interferometer for Aspherical Optical Surfaces," Laser Diagnostics, 343, 101-108 (1982).

[4] Takacs, P. Z., Qian, S., and Colbert, J., "Design of a Long-Trace Surface Profiler," Proc. SPIE 749, 59-64 (1987).

[5] Takacs, P. Z., and Qian, S., "Surface Profiling Interferometer" Associated Universities, Patent \#4,884,697, USA(1989).

[6] Susini, J., Baker, R., and Vivo, A., "Optical metrology facility at the ESRF," Rev.of Sci. Inst., 66(2), 22322234 (1995).

[7] Lammert, H., Senf, F., and Berger, M., "Improvement of Synchrotron Radiation Mirrors below the 0.1 arcsec rms Slope Error Limit with the help of a Long Trace Profiler," Proc. SPIE 3447, 168-179 (1998).

[8] Thomasset, M., Brochet, S., and Polack, F., "Latest metrology results with the SOLEIL synchrotron LTP," Proc. SPIE 5921, 592102 (2005).

[9] Thomasset, M., and Polack, F., "Characterization of optical surfaces for the present generations of synchrotron sources," Proc. SPIE 7155, 715506-12 (2008).

[10] Yashchuk, V. V., Barber, S., Domning, E. E., Kirschman, J. L., Morrison, G. Y., Smith, B. V., Siewert, F. et al., "Sub-microradian surface slope metrology with the ALS Developmental Long Trace Profiler," Nucl. Inst. and Meth. A, 616(2-3), 212-223 (2010).

[11] Geckeler, R. D., "ESAD shearing deflectometry: potentials for synchrotron beamline metrology," Proc. SPIE 6317, 63171H-13 (2006).

[12] Siewert, F., Noll, T., Schlegel, T., Zeschke, T., and Lammert, H., "The Nanometer Optical Component Measuring Machine: a new Sub-nm Topography Measuring Device for X-ray Optics at BESSY," AIP Conf. Proc. 705, 847-850.

[13] Yashchuk, V. V., Takacs, P. Z., McKinney, W. R., and Assoufid, L., "Development of a new generation of optical slope measuring profiler," The 16th Pan-American Synchrotron Radiation Instrumentation Conference, Nucl. Inst. and Meth. A, to be published, (2010).

[14] Kirschman, J. L., Domning, E. E., Franck, K. D., Irick, S. C., Mac Dowell, A. A., Mc Kinney, W. R., Morrison, G. Y. et al., "Flat-field calibration of CCD detector for long trace profiler," Proc. SPIE 6704, 67040J-11. 
[15] Yashchuk, V. V., Irick, S. C., Mac Dowell, A. A., Mc Kinney, W. R., and Takacs, P. Z., "Air convection noise of pencil-beam interferometer for long trace profiler," Proc. SPIE 6317, 63170D-12 (2006).

[16] Takacs, P. Z., and Qian, S., "Accuracy Limitations in Long-Trace Profilometry," AIP Conf. Proc. 705, 831-834 (2004).

[17] Yashchuk, V. V., "Positioning errors of pencil-beam interferometers for long trace profilers," Proc. SPIE 6317, 63170A-12.

[18] Yashchuk, V. V., "Optimal measurement strategies for effective suppression of drift errors," Rev. of Sci. Inst., 80(11), 115101 (2009).

[19] Zhao, Y., Li, Z., Li, D., Tiqiao, X., and Shaojian, X., "Principle of $\pi$-phase plate long trace profiler for synchrotron radiation optics," Opt. Comm., 200(1-6), 23-26 (2001).

[20] Qian, S., and Takacs, P., "Equal optical path beam splitters by use of amplitude-splitting and wavefrontsplitting methods for pencil beam interferometer," Proc. SPIE 5193, 79-88 (2003).

[21] Qian, S., and Takacs, P. Z., "Beam Splitter and Method for Generating Equal Optical Path Length Beams" Brookhaven Science Associates, Patent \#6,611,379, USA(2003).

[22] Qian, S., and Takacs, P., "Wave front-splitting phase shift beam splitter for pencil beam interferometer," Rev. of Sci. Inst., 74(11), 4881-4884 (2003).

[23] Anders, M., Barber, S., McKinney, W., Salmassi, F., Smith, B., and Yashchuk, V., "New LTP Phase Shifter Prototype Test Results," Light Source Beamline Note LSBL-965, Lawrence Berkeley National Lab, (2009).

[24] Takacs, P. Z., Feng, S. K., Church, E. L., Qian, S., and Liu, W.-M., "Long trace profile measurements on cylindrical aspheres," Proc. SPIE 966, 354-364 (17-19 August 1988).

[25] Irick, S. C., McKinney, W. R., Peterman, D., and Yashchuk, V. V., "Performance Test of Long Trace Profiler Part 5: Noice due to Laser Pointing Instability," Light Source Beamline Note LSBL-762, Lawrence Berkeley National Laboratory, (2005).

[26] Yashchuk, V. V., "Performance Test of Long Trace Profiler Part 5.2: Effect of Air Convection on Laser Pointing Instability," Light Source Beamline Note LSBL-766, Lawrence Berkeley National Laboratory, (2005).

[27] Anders, M., Morrison, G., and Yashchuk, V. V., "LTP Polarization Maintaining Fiber Test," Light Source Beamline Note LSBL-964, Lawrence Berkeley National Lab., (2009).

[28] Yashchuk, V. V., Irick, S. C., and Mac Dowell, A. A., "Elimination of 'ghost'-effect-related systematic errors in metrology of x-ray optics with a long trace profiler," Proc. SPIE 5858, 58580X-8.

[29] Takacs, P. Z., Qian, S., and Li, H., "Synchrotron Radiation and X-ray Mirror Metrology with the Long Trace Profiler," in [Fabrication and Testing of Aspheres], Optical Society of America, (1999).

[30] Irick, S. C., "Long trace profiler survey results," Proc. SPIE 3782, 275-282 (1999).

[31] Siewert, F., Buchheim, J., and Zeschke, T., "Characterization and calibration of 2nd generation slope measuring profiler," Nucl. Inst. and Meth. A, 616(2-3), 119-127 (2010).

[32] Rose, P., Surrel, Y., and Becker, J. M., "Specific design requirements for a reliable slope and curvature measurement standard," Measurement Science and Technology, 20(9), 095110 (2009).

[33] Siewert, F., Lammert, H., and Zeschke, T., "The Nanometer Optical Component Measuring Machine," in [Modern Developments in X-Ray and Neutron Optics], Springer Science and Business Media, (2008).

[34] Alcock, S. G., Sawhney, K. J. S., Scott, S., Pedersen, U., Walton, R., Siewert, F., Zeschke, T. et al., "The Diamond-NOM: A non-contact profiler capable of characterizing optical figure error with sub-nanometre repeatability," Nucl. Inst. and Meth. A, 616(2-3), 224-228 (2010).

[35] Yashchuk, V. V., "Universal Test Mirror for Calibration of Long Trace Profilers: Proposal," Light Source Beam Line Note LSBL-800, Lawrence Berkeley National Laboratory, (2006).

[36] Yashchuk, V. V., McKinney, W. R., Warwick, T., Noll, T., Siewert, F., Zeschke, T., and Geckeler, R. D., "Proposal for a universal test mirror for characterization of slope measuring instruments," Proc. SPIE 6704, 67040A (2007). 